TITLE:

\title{
The initial-boundary value problem for a nonlinear degenerate parabolic equation
}

AUTHOR(S):

Fukuda, Isamu; Tsutsumi, Masayoshi

CITATION:

Fukuda, Isamu ...[et al]. The initial-boundary value problem for a nonlinear degenerate parabolic equation. 数理解析研究所講究録 1989, 699: 55-73

ISSUE DATE:

1989-08

URL:

http://hdl.handle.net/2433/101469

RIGHT: 


\section{The initial-boundary value problem for \\ a nonlinear degenerate parabolic equation}

福田勇(国土館大学) IIsamu Fukuda

堤正義（早稻田大学） Masayoshi Tsutsumi

\section{Introduction and main results.}

Let $a<b$ and $\lambda>0$. We consider nonnegative solutions of the initial-boundary value problem

$$
\begin{cases}u_{t}=u_{x x}-\lambda\left|u_{x}\right|^{2} & (a<x<b, t>0) \\ u(a, t)=u(b, t)=0 & (t>0) \\ u(x, 0)=u_{0}(x) & (a<x<b)\end{cases}
$$

where initial data $u_{0}$ satisfy

(H. 1) $\quad u_{0} \in W^{1 . \infty}(a, b) \quad$ and $\quad u_{0}(x) \geqq 0 \quad(a \leqq x \leqq b)$

In order to construct a solution to the problem (1.1)-(1.3), it might be natural to employ the well-known viscosity method: Let $\varepsilon>0$ and let $u_{\varepsilon}(x, t)$ be an unique classical solution of the initial-boundary value problem for the uniformly parabolic equation:

$$
\begin{cases}\mathrm{u}_{\varepsilon \mathrm{t}}=\left(\mathrm{u}_{\varepsilon}+\varepsilon\right) \mathrm{u}_{\varepsilon \mathrm{xx}}-\lambda\left|\mathrm{u}_{\varepsilon \mathrm{x}}^{2}\right| & (\mathrm{a}<\mathrm{x}<\mathrm{b}, \mathrm{t}>0) \\ \mathrm{u}_{\varepsilon}(\mathrm{a}, \mathrm{t})=\mathrm{u}_{\varepsilon}(\mathrm{b}, \mathrm{t})=0 & (\mathrm{t}>0) \\ \mathrm{u}_{\varepsilon}(\mathrm{x}, 0)=\mathrm{u}_{0}(\mathrm{x}) & (\mathrm{a}<\mathrm{x}<\mathrm{b})\end{cases}
$$


We call u the viscosity solution of the problem (1.1)-(1.3) if $\quad u(x, t)=\lim _{\varepsilon \rightarrow 0} u(x, t)$.

Let us consider solutions with compact support and define the interface $\zeta_{ \pm}(t)$ by

$$
\zeta_{ \pm}(t)= \pm \sup \{ \pm x: u(x, t)>0\} \text { for } t>0
$$

Differentiating $u\left(\zeta_{ \pm}(t), t\right)=0$ with respect to t and using eq. (1.1), we easily see that the interface $\zeta_{ \pm}(t)$ satisfies formally

$$
\frac{d \zeta_{ \pm}(t)}{d t}=\lambda u_{x}\left(\zeta_{ \pm}(t), t\right)
$$

provided $u_{x}\left(\zeta_{ \pm}(t), t\right) \neq 0$. Thus we might expect that the support of solutions shrinks if $u_{x}\left(\zeta_{ \pm}(t), t\right) \neq 0$. Indeed, for $\lambda>\frac{1}{2}$ we have a special weak solution of the form

$$
u(x, t)=\left(T_{0}-t\right)^{\frac{1}{2 \lambda-1}}\left[c_{0}^{2}-\frac{1}{2(2 \lambda-1)} x^{2}\left(T_{0}-t\right)^{\frac{2 \lambda}{2 \lambda-1}}\right]_{+}
$$

where $\mathrm{T}_{0}$ and $\mathrm{C}_{0}$ are positive constants such that

$$
\left(-\sqrt{2(2 \lambda-1)} c_{0} \mathrm{~T}_{0}^{\frac{\lambda}{2 \lambda-1}}, \sqrt{2(2 \lambda-1)} \mathrm{c}_{0} \mathrm{~T}_{0}^{\left.\frac{\lambda}{2 \lambda-1}\right)} \mathrm{c}[\mathrm{a}, \mathrm{b}]\right.
$$

and $[\cdot]_{+}=\max (\cdot, 0)$

Apparently its support shrinks to one point. But this conjecture is not true for viscosity solutions. In [1], Bertch, Dal Passo and Ughi show that every viscosity solution of the Cauchy problem for (1.1) has a property that

$$
\operatorname{supp} u(t)=\operatorname{supp} u_{0} \quad \text { for } t>0 \text {. }
$$

It is a striking result. If $\lambda<0$, equation (1.1) is called the pressure equation, related to the porous medium equation and 
the support of solutions, spreads out as, time goes, as is suggested by the interface equation $(1.4)$.

Another curious property of eq.(1.1) is the nonuniquess phenomenon which was discovered by Dal Passo and Luckhaus. [2] $(\lambda=0)$, Ughi $[5](\lambda=0)$ and Bertch, Dal Passo and Ughi $[1](\lambda \geqq 0)$. The existence of our special weak solution u also suggests the nonuniqueness phenomenon.

We now define weak solutions of the problem (1.1)-(1.3) as follows:

Definition 1. A nonnegaive function $u \in L^{\infty}\left([0, \infty): W^{1, \infty}[a, b]\right)$ is called a weak solution of (1.1)-(1.3) if for any $T>0$

$$
u_{t} \in L^{2}([a, b] \times[0, T])
$$

and for all $t \geqq 0$

$$
\int_{a}^{b} u(x, t) \psi(x, t) d x=\int_{a}^{b} u_{0}(x) \psi(x, 0) d x
$$

$+\int_{0}^{t} \int_{a}^{b}\left\{u(x, s) \psi_{t}(x, s)-u(x, s) u_{x}(x, s) \psi_{x}(x, s)-(\lambda+1)\left|u_{x}(x, s)\right| 2 \psi(x, s)\right\} d x d t$ for any function $\psi \in C^{2}, 1([a, b] \times[0, \infty))$ with compact support in (a,b).

Note that $u \in L^{\infty}\left([0, \infty): W^{1, \infty}([a, b])\right)$ with $u_{t} \in L^{2}([a, b] \times[0, T])$ for any $T>0$ implies that $u$ is continuous in $x$ and $t$. 
In this paper we establish the global existence of (weak) solutions of (1.1)-(1.3) and investigate the uniqueness of solutions. We propose a new uniqueness class of solutions which is different from [1], [2] and [5].

As to the existence theorem, we have

Theorem 1. Let $\mathrm{u}_{0}$ satisfy (H1). Then the problem (1.1)-(1.3) has at least one weak solution.

Theorem 2. Let $\lambda>\frac{1}{2}$. Assume that $u_{0}$ satisfies (H1) and (H2) $\quad \lim _{x ! a} \frac{u_{0}(x)}{(x-a)^{2}}<\infty$ and $\quad \lim _{x \uparrow b} \frac{u_{0}(x)}{(b-x)^{2}}<\infty$.

Then u satisfies

$$
\left|u_{x x}(x, t)\right| \leqq \frac{1}{t}
$$

and, in particular, $u \in L^{\infty}\left([\delta, \infty): w^{2, \infty}([a, b])\right)$ as well as $\mathrm{u}_{\mathrm{t}} \in \mathrm{L}^{\infty}\left([\delta, \infty): \mathrm{L}^{\infty}([\mathrm{a}, \mathrm{b}])\right)$ for any $\delta>0$. Moreover, if we assume that $u_{0}$ is semiconcave, that is,

$$
\mathrm{u}_{0 \mathrm{xx}} \leqq \mathrm{C} \quad \text { in } \quad D^{\prime}
$$

for some constant C, then $u$ is also semiconcave almost everywhere, that is,

$$
u_{x x}(x, t) \leqq c \text { for a.e. }(x, t) \in[a, b] \times(0, \infty)
$$

where $C$ is also a positive constant. 
Remark 1.: In theorem 2 the hypotheses (H1) can be weakened as: follows:

( H 1$)_{\text {w }}$ $u_{0} \in L^{\infty}([a, b]), \quad u_{0}(x) \geqq 0$ a.e.

Collorary 1. Under the assumption (H1) and (H2), the problem $(1.1)-(1.3)$ has at least one weak solution which has properties in The orem 2 .

Concerning the uniqueness and continuous-dependence-on-data of solutions, we have

Theorem 3. Let $u$ and $v$ be two weak solutions coresponding to the initial data $u_{0}$ and $v_{0}$, respectively. Assume that $u$ and $v$ are semiconcave almost everywhere. Then the inequality

$$
\int_{a}^{b}|u(x, t)-v(x, t)| d x \leqq e^{c t} \int_{a}^{b}\left|u_{0}(x)-v_{0}(x)\right| d x
$$

holds valid for any $t>0$ and a positive constant c.

Corollary 2. Let $\mathrm{u}_{0}$ satisfy $(\mathrm{H} 1)_{\mathrm{w}},(\mathrm{H} 2)$ and be semiconcave. Then the problem (1.1)-(1.3) has an unique weak solution u which is also semiconcave and depends on initial data continuously in $L^{1}(a, b)$ 
Remark 2. Our special solution (1.5) is not semiconcave. Uniqueness theorem does not hold valid for the problem (1.1)-(1.3) with initial data

$$
u_{0}(x)=\left(T_{0}\right)^{\frac{1}{2 \lambda-1}}\left[C_{0}^{2}-\frac{1}{2(2 \lambda-1)} x^{2}\left(T_{0}\right)^{\frac{2 \lambda}{2 \lambda-1}}\right]+
$$

which does not satisfy (H2).

\section{Proof of Theorem 1 .}

Before proving Theorem 1, we shall obtain a priori estimates of $\quad u_{\varepsilon} \cdot$

Lemma 1. Let $\mathrm{u}_{0}$, satisfy (H1). Then

$$
\left\|\mathrm{u}_{\varepsilon}\right\|_{\mathrm{L}^{\infty}\left([0, \infty): W^{1, \infty}([a, b])\right)}
$$

and

$$
\int_{0}^{\infty} \int_{a}^{b}\left(u_{\varepsilon}(x, t)+\varepsilon\right)\left|u_{\varepsilon x}(x, t)\right|^{p-1} u_{\varepsilon x x}^{2}(x, t) d x \leqq C
$$

for any $p \geqq 1$, where and in the sequel C denotes various positive constants independent of $\varepsilon$.

Proof. The maximum principle gives

$$
0 \leqq u_{\varepsilon}(x, t) \leqq \max _{a \leqq x \leqq b} u(x)
$$


Multiplying $(1.1)_{\varepsilon}$ by $\frac{1}{p}\left(u_{\varepsilon x}(x, t) t^{p-1} u_{\varepsilon x}\right)$ and integrating by parts on $[a, b]$, we have

$$
\begin{aligned}
& \frac{1}{p(p+1)} \frac{d}{d t} \int_{a}^{b}\left|u_{\varepsilon x}\right|^{p+i} d x+\int_{a}^{b}\left(u_{\varepsilon}+\varepsilon\right)\left|u_{\varepsilon x}\right|^{p-1} u_{\varepsilon x x}^{2} d x \\
& +\frac{\lambda}{p+1}\left|u_{\varepsilon x}(a, t)\right| u_{\varepsilon x}^{p}(a, t)-\frac{\lambda}{p+1}\left|u_{\varepsilon x}(b, t)\right|{ }^{p} u_{\varepsilon x}(b, t)=0 \quad(2,4)
\end{aligned}
$$

Here and from now on we abbreviate $x$ and $t$ variables in the integrand. Since $u_{\varepsilon}$ is nonnegative, we easily see that

$$
u_{\varepsilon x}(a, t) \geqq 0 \text { and } \quad u_{\varepsilon x}(b, t) \leqq 0 \text {. }
$$

Hence integrating (2.4) from 0 to t, we obtain that, any $p \geqq 1$

$$
\begin{gathered}
\frac{1}{p(p+1)} \int_{a}^{b}: u_{\varepsilon x}{ }^{p+1} d x+\int_{0}^{t} \int_{a}^{b}\left(u_{\varepsilon}+\varepsilon\right) u_{\varepsilon x}{ }^{p-1} u_{\varepsilon x x}^{2} d x d t \\
\leqq \frac{1}{p(p+1)} \int_{a}^{b} u_{o x}{ }^{p+1} d x
\end{gathered}
$$

form which it follows that

$$
u_{\varepsilon x}(t){ }_{L}^{p+1}(a, b) \leqq u_{0 x}{ }_{L}^{p+1}(a, b) \text { for any } t>0 \text { (2.5) }
$$

and

$$
\int_{0}^{\infty} \int_{a}^{b}\left(u_{\varepsilon}+\varepsilon\right) \mid u_{\varepsilon x} \int^{p-1} u_{\varepsilon x x}^{2} d x d t \leqq c u_{0 x}{ }_{L}^{p+1}(a, b)
$$

From (2.5) we easily have (2.1). 
Lemma 2. Let $0<\varepsilon<\varepsilon_{0}$ where $\varepsilon_{0}$ is a fixed number. For any $\mathrm{T}>\mathrm{O}$,

$$
\mathrm{u}_{\varepsilon \mathrm{t}} \mathrm{L}^{2}([\mathrm{a}, \mathrm{b}] \times[0, \mathrm{~T}]) \leqq \mathrm{C}
$$

where $C$ is a positive constant independent of $\varepsilon$.

Proaf. Using $(1.1)_{\varepsilon}$ and integrating by parts, we get

$$
\begin{aligned}
& \int_{0}^{\mathrm{T}} \int_{a}^{b} u_{\varepsilon t}^{2} d x d t=\int_{0}^{T} \int_{a}^{b}\left(u_{\varepsilon}+\varepsilon\right)^{2} u_{\varepsilon x x}^{2} d x d t-\frac{2}{3} \varepsilon \lambda \int_{0}^{T}\left\{u_{x}(b, t)^{2}-u_{x}(a, t)^{2}\right\} d t \\
& +\left(\frac{2}{3} \lambda+\lambda^{2}\right) \int_{0}^{\mathrm{T}} \int_{\mathrm{a}}^{b} u_{\varepsilon \mathrm{x}}^{4} \mathrm{dxdt} \\
& \leqq\left(u_{\epsilon} L^{\infty}([a, b] \times[0, T])^{+\varepsilon}\right) \int_{0}^{T} \int_{a}^{b}\left(u_{\varepsilon}+\varepsilon\right) u_{\varepsilon x x}^{2} d x d t \\
& +\frac{4}{3} \varepsilon \lambda \mathrm{T} \mathrm{u}_{\varepsilon \mathrm{X}} \|_{\mathrm{L}}^{3}([\mathrm{a}, \mathrm{b}] \times[0, \infty))+\left(\frac{2}{3} \lambda+\lambda^{2}\right)(\mathrm{b}-\mathrm{a}) \mathrm{T} \mathrm{u}_{\varepsilon \mathrm{X}} \mathrm{L}^{\infty}([\mathrm{a}, \mathrm{b}] \times[0, \infty))
\end{aligned}
$$

From (2.1) and (2.2) with $p=1$, we can easily obtain (2.7).

Proof of Theorem 1. From (2.1), (2.3) and (2.7), we see that there exists a nonnegative function $u \in L^{\infty}\left([0, \infty): C([a, b]) \cap W^{1, \infty}[a, b]\right)$ with $u_{t} \in L^{2}([a, b] \times[0, T])($ for any $T>0)$ and we can extract a subsequence of $\left\{u_{\varepsilon}\right\}$, which is denoted by $\left\{u_{\varepsilon_{i}}\right\}$, such that, as $\varepsilon_{\mathrm{i}} \longrightarrow 0$, 
and

$$
\begin{aligned}
& u_{\varepsilon i} \longrightarrow u \text { strongly in } c([a, b] \times[0, T]) \\
& u_{\varepsilon_{i} x} \longrightarrow u_{x} \text { weakly star in } L^{\infty}([a, b] \times[0, \infty))
\end{aligned}
$$

$$
\mathrm{u}_{\varepsilon_{\mathrm{i}} \mathrm{t}} \longrightarrow \mathrm{u}_{\mathrm{t}} \quad \text { weakly in } \mathrm{L}^{2}([\mathrm{a}, \mathrm{b}] \times[0, \mathrm{~T}])
$$

In order to show that $u$ is a weak solution of (1.1)-(1.3), it suffices to show that, for any $T>0$

$$
\left.\left|u_{\varepsilon_{i} x}\right|^{2} \longrightarrow \operatorname{lu}_{x}\right|^{2} \quad \text { in } L^{1}([a, b] \times[0, T])
$$

and this implies

$$
u_{\varepsilon_{i} x} \longrightarrow u_{x} \text { strongly in } L^{2}([a, b] \times[0, T])
$$

From (2.1) and (2.2), we have

$$
\left(\mathrm{u}_{\varepsilon}^{2}\right) \mathrm{xx}_{\mathrm{L}}{ }^{2}([\mathrm{a}, \mathrm{b}] \times[0, \mathrm{~T}]) \leqq 2 \mathrm{u}_{\varepsilon} \mathrm{u}_{\varepsilon \mathrm{xx}}+\mathrm{u}_{\varepsilon \mathrm{x}}^{2} \mathrm{~L}_{([\mathrm{a}, \mathrm{b}] \times[0, \mathrm{~T}])} \leqq C .
$$

We also have

$$
\left(u_{\varepsilon}^{2}\right) x t_{L^{2}}^{2}\left(0, T: H^{-1}(a, b)\right) \leqq C\left(u_{\varepsilon}^{2}\right) t^{2}([a, b] \times[0, T]) \leqq C .
$$

By virtue of Aubin's compactness theorem (see J.L.Lions [4]), we may assume that

$$
\left(u_{\varepsilon_{i}}^{2}\right)_{x}=2 u_{\varepsilon_{i}} u_{\varepsilon_{i} x} \longrightarrow 2 u_{x}=\left(u^{2}\right)_{x} \quad \text { strongly in } L^{2}([a, b] \times[0, T]) .
$$

Hence we may also assume that

$$
u_{\varepsilon_{i}}^{u_{\varepsilon_{i}}} \longrightarrow u_{x}^{u} \quad \text { a.e. in }[a, b] \times[0, \infty)
$$

from which it follows that

$$
\mathrm{u}_{\varepsilon_{\mathrm{i}} \mathrm{x}} \longrightarrow \mathrm{u}_{\mathrm{x}} \quad \mathrm{a} . \mathrm{e} \text {. in } \quad[\mathrm{a}, \mathrm{b}] \times[0, \infty)
$$


since $\quad \frac{\partial u}{\partial x}=0$ a.e. in $E=\{x \in[a, b] ; u=0\} \quad$ (s ee Kinder lehrer-Stampacchia [3], p53) and

$$
u_{\varepsilon_{i} x} \longrightarrow u_{x} \text { a.e. in }{ }^{c} E=\{x \in[a, b] ; u>0\}
$$

In view of Lebesgue's bounded convergence theorem we can easily abtain

$$
\lim _{m \rightarrow \infty} \int_{0}^{T} \int_{a}^{b}\left|u_{m x}^{2}\right| d x d t=\int_{0}^{T} \int_{a}^{b}\left|u_{x}^{2}\right| d x d t .
$$

On the other hand, from (2.1) we may assume that $u_{m x}$ converges to $u_{x}$ weakly in $L^{2}([a, b] \times[0, T])$. Hence

$$
u_{m x} \longrightarrow u_{x} \quad \text { strongly in } L^{2}([a, b] \times[0, T])
$$

This completes the proof of Theorem 1 .

\section{Proof of Theorem 2 .}

Lemma 3. Let $\mathrm{u}_{0}$ satisfy (H1) and (H2). Then, for any

$\mathrm{t}>0$

$$
\left|u_{\varepsilon}(a, t)\right| \leqq \sqrt{\varepsilon} C
$$

and

$$
\left|u_{\varepsilon}(b, t)\right| \leqq \sqrt{\varepsilon} \mathrm{C}
$$


Proof. We oniy show that (3. hold valid. From (HZ) we see that for some $\delta>0$ and $C_{1}>0$

$0 \leqq u_{0}(x) \leqq C_{1}\left\{(x-a)^{2}+\sqrt{\varepsilon}(x-a)\right\}$ for an $y \quad x \in(a, a+\delta)$

Let $T>0$ be fixed. For any $(x, t) \in[a, a+\delta] \times[0, T]$ set

$$
\bar{u}(x, t)=A\left\{(x-a)^{2}+\sqrt{\varepsilon}(x-a)\right\}
$$

where $A$ is chosen so large that

$$
\mathrm{A} \geqq \mathrm{C}_{1}
$$

and

$$
A\left(\delta^{2}+\sqrt{\varepsilon} \delta\right) \geqq \max _{\substack{a \leqq x \leqq a \\ 0 \leqq t \leqq T}} u_{\varepsilon}(x, t)
$$

Sote that $u \in C^{2,1}((a, b) \times[0, T])$. Direct calculation gives

$$
\begin{aligned}
& \bar{u}_{t}-(\bar{u}+\varepsilon) \bar{u}_{x x}+\lambda\left(\bar{u}_{x}\right)^{2} \\
& =2(2 \lambda-1) A^{2}(x-a)^{2}+2(2 \lambda-1) A^{2} \sqrt{\varepsilon}(x-a)+2 \varepsilon A(\lambda A-2) \\
& \geqq 0 \quad \text { in }(a, a+\delta) \times(0, T)
\end{aligned}
$$

provided that $\lambda \geqq \frac{1}{2}$ and that $A$ is so large that.

$$
\lambda A-2>0
$$

By virtue of $(3.3)-(3.7)$ we apply the maximum principle to

obta in

$$
0 \leqq u_{\hat{\varepsilon}}(\mathrm{x}, \mathrm{t}) \leqq \overline{\mathrm{u}}(\mathrm{x}, \mathrm{t}) \quad \text { in } \quad[\mathrm{a}, \mathrm{a}+\delta] \times[0, \mathrm{~T}] .
$$

Hence

$$
0 \leqq u_{\varepsilon}(a, t)=\lim _{h} \prod_{0} \frac{u_{\varepsilon}(a+h, t)-u_{\varepsilon}(a, t)}{h} \leqq \lim _{h} \frac{u(a+h)}{h}=A \sqrt{\varepsilon} .
$$

Thus we have (3.1). 
Lemma 4 . Inder the same assumption

$$
\left|u_{\varepsilon \times x}\right| \leqq \frac{c}{t} \quad \text { for ant } t>0 \text {. }
$$

Moreover, if $u_{0 x x} \leqq C_{2}$ then

$$
u_{\varepsilon \times x} \leqq C_{3}
$$

where $c_{3}$ is a constant.

$$
\begin{aligned}
& \text { Proof. } \quad \text { Putting } \quad p=\frac{u_{\varepsilon t}}{u_{\varepsilon}+\varepsilon} \text {, we have } \\
& p_{t}=\left(u_{\varepsilon}+\varepsilon\right) p_{x x}+2(1-\lambda) u_{\varepsilon x} p_{x}+p^{2} \quad(x, t) \equiv(a, b) \times(0, \infty) \\
& p(a, t)=p(b, t)=0 \quad t \equiv(0, \infty) \\
& p(x, 0)=u_{0 x x}-\frac{a_{0 x}^{2}}{u_{0}+\varepsilon} \quad x \in(a, b)
\end{aligned}
$$

The standard comparison theorem yields that

$$
p \geqq-\frac{1}{t}
$$

Esing $(1.1)_{\varepsilon}$, we easily see that

$$
u_{\varepsilon x X} \geqq-\frac{1}{t}
$$

We put $q=u_{\varepsilon x x}$ to obtain that

$$
q_{t}=\left(u_{\varepsilon}+\varepsilon\right) q_{x x}+2(1-\lambda) u_{\varepsilon x} q_{x}+(1-2 \lambda) q^{2}
$$

As for the boundary conditions, we utilize (1.1) $)_{\varepsilon}$ to get 


$$
q(a, t)=\frac{a}{\varepsilon}\left|u_{\varepsilon x}(a, t)\right|^{2}, \quad q(b, t)=\frac{\lambda}{\varepsilon}\left|u_{\varepsilon x}(b, t)\right|^{2}
$$

for any $t>0$. In view of Lemma 3 , we see that

$$
0 \leqq q(a, t) \leqq a c^{2}, \quad 0 \leqq q(b, t) \leqq a c^{2}
$$

Hence the comparison theorem yields that, if $\lambda>\frac{1}{2}$

$$
q(x, t)=u_{\varepsilon x x}(x, t) \leqq \frac{C}{(2 \lambda-1)}
$$

for some constant $C>0$.

$$
\begin{aligned}
& \text { if } u_{0 x x} \leqq C_{2}, \quad \geqq \frac{1}{2} \text { and }(3.11)-(3.13) \text { yield that } \\
& u_{\varepsilon x x}(x, t) \leqq C_{3}
\end{aligned}
$$

where $C_{3}=\max \left(\lambda C^{2}, C_{2}\right)$ is independent of $\hat{\varepsilon}$.

Proof of Theorem 2. Because of Lemma 4 , we see that $\left\{u_{\varepsilon x x}\right\}$ is bounded in $L^{\infty}([a, b] \times[\delta, \infty))$ for every $\delta>0$. Hence we can assume that

$$
u_{\varepsilon_{i} x x} \longrightarrow u_{x x} \quad \text { weakly star in } L^{\infty}([a, b] \times[\delta, \infty))
$$

and

$$
\left|u_{x x}(x, t)\right| \leqq \frac{C}{t} \quad \text { for any }(x, t) \in[a, b] \times[\delta, \infty)
$$

If $u_{0 x x} \leqq \tau$, from (3.14) we have

$$
u_{x x}(x, t) \leqq C \quad \text { for any }(x, t) \in[a, b] \times[0, \infty)
$$

This completes the proof of Theorem 2 . 


\section{Proof of Thearem 3.}

Let $u$ and $v$ be two weak soluions of $(1.1)-(1.3)$ with initial data $u_{0}$ and ${ }_{0}$, respectively. Let $T>0$ be fixed and put $w(x, t)=u(x, t)-v(x, t)$ and $w_{0}(x)=u_{0}(x)-v_{0}(x)$.

Then we have

$$
\begin{aligned}
& \int_{a}^{b} w(x, T) \psi(x, T) d x=\int_{a}^{b} w_{0}(x) \psi(x, 0) d x \\
& +\int_{0}^{T} \int_{a}^{b}\left\{w \psi_{t}-\left(u_{x}-v v_{x}\right) \psi_{x}-(\lambda+1)\left(\left|u_{x}\right|^{2}-\left.v_{x}\right|^{2}\right) \psi\right\} d x d t
\end{aligned}
$$

for any $\psi \in C^{2}, 1([a, b] \times[0, \infty))$ with compact support in $(a, b)$.

For each $n \in N$ define

$$
g_{n}(x)=\left\{\begin{array}{ccc}
1 & \text { if } & \frac{1}{n}<s \\
n s & \text { if } & |s| \leqq \frac{1}{n} \\
-1 & \text { if } & s<-\frac{1}{n}
\end{array}\right.
$$

and

$$
\Psi=\left\langle g_{\mathrm{n}}\left(\left(\mathrm{u}^{2}-\mathrm{v}^{2}\right) \theta_{\mathrm{k}} \theta_{\mathrm{m}} \star \rho_{\nu} \star \sigma_{\mu}\right) \star \rho_{i} \star \sigma_{\mu}\right\} \theta_{\mathrm{k}} \theta_{\mathrm{m}}
$$

where $\rho_{y}$ and $\sigma_{\text {il }}$ are the standard molifiers with respect to $x$ and $t$, respectively; $\theta_{k}\left(\frac{x}{k}\right)$ where $\theta \in C_{0}^{\infty}((a, b))$ with $0 \leqq \theta \leqq 1$ and $\theta(x)=1$ in a neighborhood of 0 (we may assume $0 \in(a, b)$ ) and 
69

$$
\begin{aligned}
& \otimes_{m}(t) \in C_{0}^{\infty}((0, \infty)) \text { such that } 0 \leqq \theta_{m} \leqq 1 \text { and } \theta_{m}(t) \text { tends to the } \\
& \text { indicator function of }\left[s_{1}, s_{2}\right]\left(0<s_{1}<s_{2}\right) \text { as } m \rightarrow \infty \text {. Then } \\
& \Psi \in C_{0}^{\infty}((a, b) \times(0, \infty)) \text { and } \Psi(x, t) \geqq 0 \text { for any }(x, t) \in(a, b) \times(0, \infty) .
\end{aligned}
$$

Substituting $\Psi$ for a test function $\psi$ in ( 3.1$)$, we have

$$
\int_{0}^{\mathrm{T}} \int_{\mathrm{a}}^{\mathrm{b}}\left\{w \Psi_{\mathrm{t}}-\left(\mathrm{uu_{ \textrm {x } }}-\mathrm{vv}_{\mathrm{x}}\right) \Psi_{\mathrm{x}}-(\lambda+1)\left(\left|\mathrm{u}_{\mathrm{x}}\right|^{2}-\left|\mathrm{v}_{\mathrm{x}}\right|^{2}\right) \Psi\right\} \mathrm{dxdt}
$$

From ${ } \in L^{2}([a, b] \times[0, T])$ for any $T>0$ and $\Psi \in C_{0}^{\infty}((a, b) \times(0, \infty))$ we

get

$$
\int_{0}^{\mathrm{T}} \int_{a}^{b} w \Psi_{t} d x d t=-\int_{0}^{\mathrm{T}} \int_{a}^{b} w_{t} \Psi d x d t
$$

Letting $y$ and $\mu$ tend to infinity, we can easily see that

$$
\begin{aligned}
& I_{1}(k, m, n)-I_{2}(k, m, n)-I_{3}(k, m, n) \\
& \equiv \int_{0}^{\mathrm{T}} \int_{a}^{b} w_{t}{ }^{\theta} k^{\theta}{ }_{m}^{g}{ }_{n}\left(\left(u^{2}-v^{2}\right){ }^{\theta} k^{0}{ }_{m}\right) d x d t \\
& -\left[-\int_{0}^{T} \int_{a}^{b}\left(u u_{x}-v v_{x}\right)\left\{g_{n}\left(\left(u^{2}-v^{2}\right){ }^{a} k_{m}{ }_{m}\right)\right\} x d x d t\right]
\end{aligned}
$$

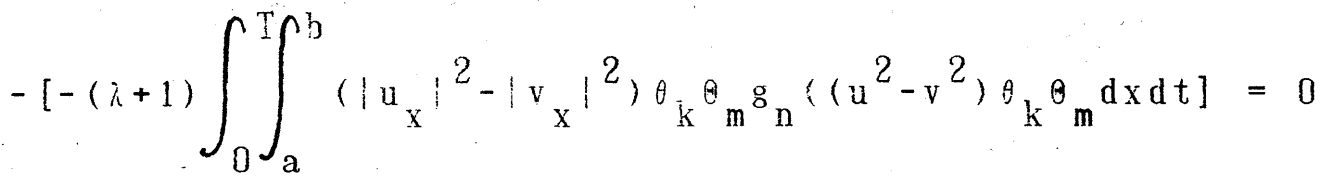

$-15-$ 
As $n$ tends to infinity, we find $I_{1}(k, m, n)$ tends to

$$
\tilde{I}_{1}(k, m)=\int_{0}^{T} \int_{a}^{b} w_{t} \theta_{k} \theta_{m} \operatorname{sgn}\left((u-v) \theta_{k}{ }_{m}\right) d x d t
$$

since $\operatorname{sgn}\left(\left(u^{2}-v^{2}\right) \theta_{k} \theta_{m}\right)=\operatorname{sgn}\left((u-v) \theta_{k} \theta_{m}\right)$.

Moreover, $\mho_{m}(t)=0$ near 0 and $T$, then we have

$$
\begin{aligned}
\tilde{I}_{1}(k, m) & =\int_{0}^{T} \int_{a}^{b}\left(\left|w \theta_{k} \theta_{m}\right|\right) d x d t-\int_{0}^{T} \int_{a}^{b}\left|w \theta_{k}\right|\left(\theta_{m}\right)_{t} d x d t \\
& =-\int_{0}^{T} \int_{a}^{b}{ }^{\theta}{ }_{k} \mid\left(\theta_{m}\right)_{t} d x d t
\end{aligned}
$$

As for $I_{2}(k, m, n)$, using chain rule, we get

$$
\begin{aligned}
& I_{2}(k, m, n)=-2 \int_{0}^{\mathrm{T}} \int_{a}^{b}\left(u u_{x}-v v_{x}\right)^{2} g_{n}^{\prime}\left(\left(u^{2}-v^{2}\right){ }^{\theta}{ }_{k}{ }^{\theta}{ }_{m}\right){ }^{\theta} k^{\theta}{ }_{m} d x d t \\
& -\int_{0}^{T} \int_{a}^{b}\left(u u_{x}-v v_{x}\right) g_{n}^{\prime}\left(\left(u^{2}-v^{2}\right) \theta_{k} \theta_{m}\right)\left(u^{2}-v^{2}\right) \theta_{m}^{2} \theta_{k}\left(\theta_{k}\right) d x d t \\
& -\int_{0}^{T} \int_{a}^{b}\left(u_{x}-v v_{x}\right) g_{n}\left(\left(u^{2}-v^{2}\right) \theta_{k} \theta_{m}\right) \theta_{m}^{2}{ }_{k}\left(\theta_{k}\right) d x d t
\end{aligned}
$$


Since the first term onthe right hand side is nonpositive and $\left(\theta_{k}\right) \leqq \frac{C}{k}$, we have

$$
I_{2}(k, m, n) \leqq \frac{c}{k}\left(u_{L^{3}}^{3}+v L_{L^{\infty}}^{3}+u_{L^{\infty}}+v_{L^{\infty}}\right)\left(u_{x_{L}}{ }^{+} v_{x_{L}}{ }^{2}\right)
$$

where $L^{p}=L^{p}([a, b] \times[0, T]) \quad(p=2, \infty)$. Since $u L^{\infty}, v L_{L}^{\infty}, u_{x}{ }_{L}^{2}$ and $v_{x}{ }^{2}$ are bounded, we get

$$
I_{2}(k, m, n) \leqq \frac{C}{k}
$$

where $C$ depends on $u L_{L}^{\infty}, v_{L}^{\infty}, u_{x_{L}}{ }^{\infty}$ and $v_{x}{ }_{L}$

$$
\text { Since } \operatorname{sgn}\left(\left(u^{2}-v^{2}\right){ }^{6} k_{m}^{8}\right)=\operatorname{sgn}\left(w \theta_{k}{ }_{m}\right), \text { letting } n \longrightarrow \infty
$$

we see that $I_{3}(k, m, n)$ tends to

$$
\tilde{I}_{3}(k, m)=-(\lambda+1) \int_{0}^{T} \int_{a}^{b}\left(\left|u_{x}\right|^{2}-\left|v_{x}\right|^{2}\right) \theta_{k} \theta_{m} \operatorname{sgn}\left(w \theta_{k} \theta_{m}\right) d x d t
$$

Recalling that $u_{x x}$ and $v_{x x}$ are semiconcave, we have

$$
\begin{aligned}
& \widetilde{I}_{3}(k, m)=-(\lambda+1) \int_{0}^{T} \int_{a}^{b}\left(i w \theta_{k} \theta_{m}\right)_{x}\left(u_{x}+v_{x}\right) d x d t \\
& -(i+1) \int_{0}^{T} \int_{a}^{b}(u-v)\left(u_{x}-v_{x}\right)\left(\theta_{k}\right){ }_{x}^{\theta}{ }_{m} \operatorname{sgn}\left(w \theta_{k} \theta_{m}\right) d x d t
\end{aligned}
$$




$$
\begin{aligned}
& \leqq(i+1) \int_{0}^{\mathrm{r}} \int_{a}^{b}\left|w_{k}{ }_{k} \theta_{m}\right|\left(u_{x x}+v_{x x}\right) d x d t \\
& +(a+1) \int_{0}^{T} \int_{a}^{b}(|u|+|v|)\left(\left|u_{x}\right|+\left|v_{x}\right|\right)\left|\left(\theta_{k}\right){ }_{x}\right| d x d t \\
& \leqq c \int_{0}^{T} \int_{a}^{b}\left|w \theta_{k} \theta_{m}\right| d x d t+\frac{c}{k} .
\end{aligned}
$$

Hence eq. (4.3) with (4.5), (4.6) and (4.7), implies that

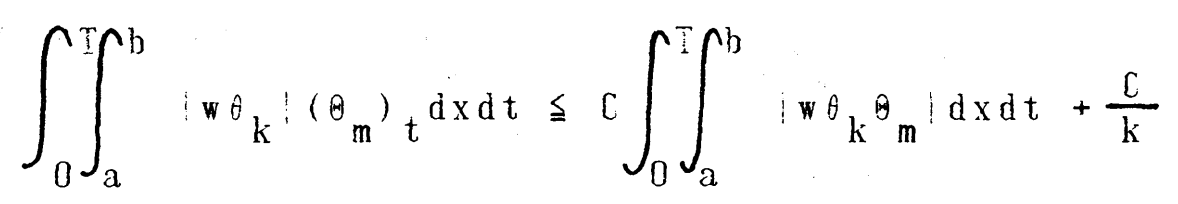

In (4.8) letting $\mathrm{k}, \mathrm{m} \longrightarrow \infty$, we find that

$$
\int_{a}^{b}\left|w\left(x, s_{2}\right)\right| d x-\int_{a}^{b}\left|w\left(x, s_{1}\right)\right| d x \leqq c \int_{s_{1}}^{s} 2 \int_{a}^{b}|w(x, s)| d x d s
$$

for any $s_{1}$ and $s_{2}\left(0<s_{1}<s_{2}\right)$.

As $s_{2}=t$ and $s_{1}$ tends to 0 , we have

$$
\int_{a}^{b}|(x, t)| d x-\int_{a}^{b} i w_{0}(x)\left|d x \leqq c \int_{0}^{t} \int_{a}^{b} i w(x, s)\right| d x d s
$$

from which it follows that, for any $t \geq 0$ 


$$
\int_{a}^{b}|w(x, t)| d x \leqq e^{c t} \int_{a}^{b} w_{0}(x) \mid d x
$$

This completes the proof of Theorem 3 . Corollary 2 is easily obtained from (4.9).

\section{$R E F E E \mathbb{N} \mathbb{E} \mathbb{E}$}

[1] BERTSCH M., DAL PASSO R. \& UGHI M., Nonuniqueness and irregularuty results for a nonlinear degenerate parabolic equation, "Nonlinear Diffusion Equations and Their Equilibrium States I" Ed. by W.M.Ni, L.A.Peletier \& J.Serrin, Springer-Verlag (1986) [2] DAL PASSO R. \& LUCKHAUS S., A degenerate diffusion problem noy in divergence form, J.Diff.Eq.69,1 (1987)

3] KINDERLEHRER D. \& STAMPACCHIA G., An introduction to variational inequalities and their applications, Academic Press (1980)

[4] LIONS J.L., Quelques Méthodes de Résolution des Problèmes aux Limites Non Linéaires, Dunod Gauthier-Villars (1969)

[5] UGHI M., A degenerate parabolic equation modeling the spread of an epidemic, Annali. Math. Pure Appl.,143,385 (1986) 